\title{
Experiência em pacientes com suspeita de hepatopatia crônica e contra-indicação para biópsia hepática percutânea utilizando a agulha de Ross modificada
}

\author{
A. C. Maciel, S. G. Silva de Barros, D. P. Tarasconi, L. C. V. Severo únior, C. T. S. Cerski, D. de O. I lha
}

Serviço de Radiol ogia da I rmandade da Santa Casa de Misericórdia de Porto Alegre e Serviço de Patol ogia do Hospital de Clínicas de Porto Alegre, RS.

Resumo - Овј етіvos. O trabalho visou introduzir em nosso meio a técnica de biópsia hepática transjugular orientada por métodos radiológicos para a obtenção do diagnóstico histológico em pacientes clínica e laboratorialmente diagnosticados com hepatopatia crônica e com pelo menos uma das contra-indicações à biópsia hepática percutânea: coagulopatia, ascite maciça e anemia crônica acentuada com insuficiência renal crôni ca ou obesidade mórbida.

MÉtodos. Biópsia hepática aspi rativa foi obtida com agulha de Ross modificada, através da punção da veia jugular interna com cateterização da veia hepática direita sob controle fluoroscópico.

Resultados. Trinta e nove pacientes foram estudados, obtendo-se tecido hepático em 32 (82\%) sendo satisfatório para diagnóstico histológico em 25 (64,1\%), com concordância entre o diagnóstico pré e pós-biópsia em 11 (28,2\%) e discordância em 14 pacientes (35,9\%). O procedimento foi bem tolerado pela maioria dos pacientes; contudo um paciente apresentou sangramento retroperitoneal, necessitando cirurgia imediata para controle da hemorragia.

Conclusões. A biópsia hepática transjugular é um método diagnóstico úti l para o estudo histopatológico na suspeita de hepatopatia crônica com contra-indicações a biópsia hepática percutânea. Em nossa série foi obtido diagnóstico histopatológico em 64,1\% dos indivíduos estudados. Pacientes com suspeita clínica de cirrose tiveram baixo diagnóstico histopatológico (50\%) quando comparados com indivíduos sem essa suspeita (78,9\%). O método é relativamente complexo, podendo apresentar complicações graves, e deve ser realizado em centros de excelência em radiologia e hepatologia.

UNITERMOS: Biópsia hepática. Biópsia hepática Transjugular. Coagulopatia. Hepatopatia crônica. Diagnóstico.

\section{INTRODUÇÃO}

A necessi dade de um adequado diagnóstico das hepatopatias crônicas levou vários autores a desenvolverem técnicas alternativas de biópsia do fígado. O diagnóstico histopatológico pode indicar a etiol ogia, a ati vi dade e o estadiamento da doença hepática, com conseqüente mudança na conduta médica.

A biópsia hepática pode ser realizada através de quatro métodos: ${ }^{1}$ biópsia hepática percutânea (BHP), às cegas; biópsia hepática durante laparoscopia ou laparotomia; biópsia hepática percutânea guiada por ultra-sonografia ou tomografia computadorizada; biópsia hepática transjugular (BHTJ).

Foi em 1964 que Dotter relatou com sucesso as primeiras $\mathrm{BHT}$ J feitas a partir de estudos experimentais em cães². Em 1967, Hanafee e Weiner publicaram a primeira descrição de cateterismo transjugular de seres humanos para a realização de col angiografia ${ }^{3}$. Em 1970, estes últimos autores fizeram, em alguns pacientes, as primeiras BHTJ de uma série, cuja finalidade foi a realização de colangiografia transvenosa ${ }^{4}$. Em 1973, Rösch et al., Oregon University ${ }^{5,6}$, descreveram a primeira grande série, com relato de $44 \mathrm{BHTJ}$, tendo o procedimento sido associado à colangiografia em alguns pacientes.

Os benefícios da biópsia hepática são muitos. As facilidades de realização, assim como as infreqüentes complicações e a importância diagnóstica, têm incrementado seu uso7. A biópsia hepática transjugular assume importância especial sempre que a biópsia percutânea ofereça grandes riscos para complicações, sendo os principais riscos a presença de distúrbi os de coagulação, ascite maciça, anemia crônica acentuada associada à insuficiência renal crônica com uso de anticoagulantes (heparina) e obesi dade mórbi da. A escol ha do método de biópsia hepática dependerá das condições do paciente, da disponibilidade de material e de pessoal treinado. Entretanto, os limites mínimos 
recomendáveis nas alterações de coagulação para a escol ha do método de biópsia hepática variam de acordo com diferentes autores-11.

O objetivo do trabalho foi introduzir em nosso meio a técnica de biópsia hepática transjugular (BHTJ ) realizada através da fluoroscopia para a obtenção do diagnóstico histopatológico em pacientes clínica e laboratorial mente diagnosticados com hepatopatia crônica e contra-indicações à biópsia hepática percutânea (BHP).

\section{CASUÍSTICA E MÉTOdo}

Foram selecionados apenas pacientes adultos no período de março de 1994 a junho de 1996. Indicou-sea BHTJ quando os pacientes apresentavam pelo menos uma das contra-indicações reconhecidas à biópsia hepática percutânea: 1. Coagulopatia, verificada por al terações em, pelo menos, um dos seguintes testes - tempo de protrombina (menor do que $60 \%$ do controle), tempo parcial de tromboplastina (5 segundos acima do controle) e/ ou contagem de plaquetas (menor do que 60.000 / $\mathrm{mm}^{3}$ ); 2. Ascite maciça (parede abdominal tensa com ascite confirmada por ecografia e não responsiva à tratamento com diuréticos); 3 . Anemia crônica acentuada associada à insuficiência renal crônica, com hematócrito igual ou inferior a $29 \%$ e uso de anti-coagulantes (heparina); 4. Obesidade mórbida (peso corporal $20 \%$ acima do peso ideal).

O diagnóstico clínico ou pré-biópsia foi baseado na impressão clínica e na utilização de métodos complementares e foi elaborado por hepatologistas, clínicos gerais, hematologistas, gastroenterologistas e nefrologistas pertencentes aos serviços do hospital. Os diagnósticos clínicos foram: provável cirrose em 20 pacientes (51,3\%) (etiologia alcoólica em 10; HCV em oito; HBV em um; e biliar secundária em um); hepatites crônicas em 13 pacientes (33,3\%) (HCV em sete; HBV em dois; e HCV e HBV, simultaneamente em quatro); hepatite al coólica em três pacientes (7,6\%), hemocromatose em um paciente (2,5\%); hepatite por citomegalovírus em um $(2,5 \%)$ e tumor metastático, infiltrativo em um $(2,5 \%)$ paciente. Para determinar possível etiologia viral, o soro de todos os pacientes foi testado para a presença de anti-HCV de segunda geração (considerado positivo somente quando reagente em um segundo teste após um período de 30 dias) e para a presença de $\mathrm{H}$ bsAg e Anti-HBs testados pel o método MEIA-AXSYM (Laboratório Abbott).

Os exames foram realizados no Serviço de Hemodinâmica do Pavilhão São J osé, da I rmandade da Santa Casa de Misericórdia de Porto Alegre (ISCMPA), e as biópsias foram enviadas para o

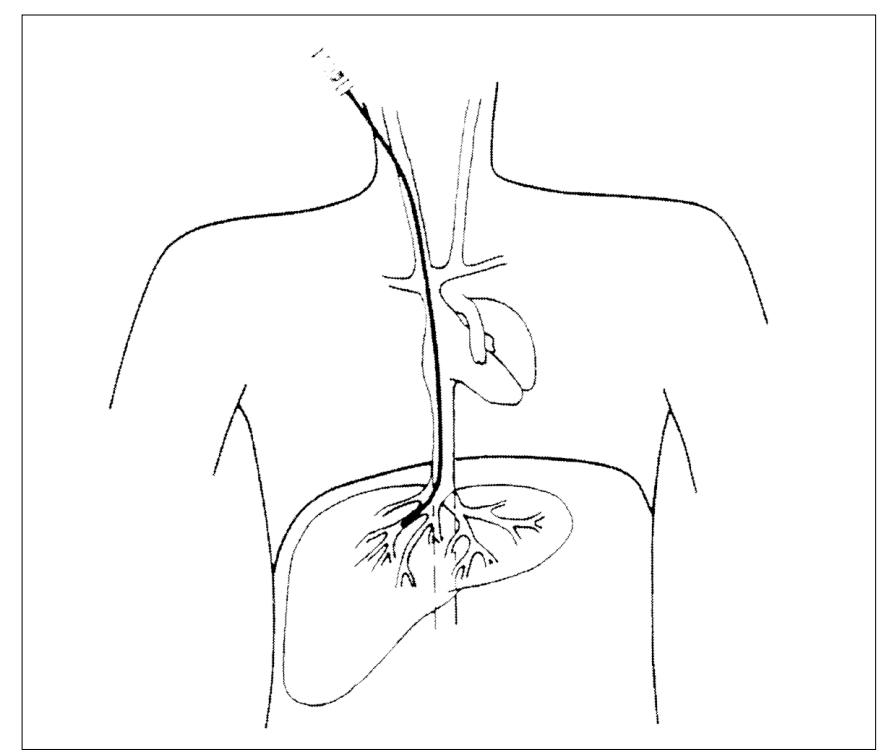

Fig. 1 - Esquema do trajeto percorrido pelo fio guia, cateter e agulha de biópsia, cuja extremi dade encontrase na vei a hepática direita (F onte: Zwi ebel et al ., 1993).

Serviço de Patologia do Hospital de Clínicas de Porto Alegre (HCPA). Os paci entes for am informados do procedimento, de seus benefícios e de eventuais complicações; todos assinaram termo de consentimento informado previamente e aprovado pela Comissão de Ética das instituições envolvidas. O equipamento radiológico utilizado para os exames foi móvel, com arco em "C" ou "arco cirúrgico", com intensificador de imagens, monitor de vídeo, memória e subtração digital (modelo Siremobil-2, Siemens). Para o procedimento utilizouse a fluoroscopia e efetuaram-se registros em radiografias digitais. A fluoroscopia usada oscilou em torno de $90 \mathrm{kV}$ e 1,5 mA. A filtração inerente é de $3 \mathrm{~mm}$ de equivalência de Alumínio (segundo o manual do aparel ho). Os val ores máximos de taxa de dose e dose absorvida foram determinados a partir de medidas realizadas com monitor de radiação 660, câmara de ionização 660-3, marca Victor een pelo Serviço de Física Médica do HCPA.

O procedimento foi realizado após oito horas de jejum, acompanhado por anestesista e monitorização el etrocardiográfica. A veia jugular interna direita (VJ ID) foi utilizada em todos os pacientes submetidos BHTJ, na localização supraclavicular, pela técnica de Sel dinger. Sob controle fluoroscópico, com a utilização de fio guia e cateteres, foi feita a cateterização da veia hepática direita. Todas as biópsias hepáticas foram obtidas no lobo direito do fígado devido ao seu maior tamanho e ao ângulo agudo que a veia hepática di reita faz com a veia cava inferior. Usou-se para a biópsia a agulha de Ross modificada, pela técnica aspirativa de Menghini ${ }^{12}$. A Fig. 1 demonstra o trajeto percorrido pela agulha. 


\begin{tabular}{|c|c|c|}
\hline \multicolumn{3}{|c|}{$\begin{array}{l}\text { Tabela } 1 \text { - Indicações para BHTJ na suspeita de hepatopatia } \\
\text { crônica }(n=39)\end{array}$} \\
\hline Indicações & $\mathbf{N}^{\circ}$ Pacientes & $\%$ Pacientes \\
\hline Coagulopatia isolada & 13 & $33,3 \%$ \\
\hline Coagulopatia e ascite & 17 & $43,6 \%$ \\
\hline $\begin{array}{l}\text { Coagulopatia, ascite } \\
\text { e obesidade mórbida }\end{array}$ & 1 & $2,6 \%$ \\
\hline Coagulopatia e obesidade mórbida & 1 & $2,6 \%$ \\
\hline $\begin{array}{l}\text { Anemia crônica } \\
\text { (hematócrito = ou < 29\%) }\end{array}$ & 7 & $17,9 \%$ \\
\hline Total & 39 & $100 \%$ \\
\hline
\end{tabular}

*Pacientes com insuficiência renal crônica em hemodiálise com uso de heparina.

Tabela 2 - Concordância entre o diagnóstico clínico (prébiópsia) e histopatológico (pós-biópsia) $(n=11)$

\begin{tabular}{|ll|}
\hline $\begin{array}{l}\text { Diagnóstico pré-biópsia } \\
\text { (no-pacientes) }\end{array}$ & $\begin{array}{l}\text { Diagnóstico pós-biópsia } \\
\text { (no-pacientes) }\end{array}$ \\
Cirrose (4) & Cirrose (4) \\
Hepatite crônica (3) & Hepatite crônica (3) \\
Hepatite alcoólica (3) & Esteatoepatite (3) \\
Hemocromatose (1) & Hemocromatose (1) \\
\hline
\end{tabular}

Concluídas as biópsias, retirou-se o cateter, efetuando-se a compressão da região cervical por cinco minutos. Os pacientes permaneceram com a cabeceira da cama elevada e monitorização dos sinais vitais durante três horas.

Os espéci mens foram medidos e a comparação dos seus comprimentos entre os diferentes grupos de diagnósticos histológicos obtidos foi feita pelo teste de Wilcoxon-Mann-Whitney $(\mathrm{WMW})^{13}$, pois a distribuição dessas variáveis, em alguns casos, não foi gaussiana. O nível de significância usado foi de 0,05 .

No diagnóstico histopatológico de hepatite mínima e hepatite crônica, com suas respectivas graduação e estadiamento, foram utilizados os critérios de Bianchi e Gudat ${ }^{14}$. Estes critérios, associados à evidência de hepatite viral C ou B, consistem na identificação de um discreto infiltrado linfocitário em apenas al guns espaços-porta, acompanhados por focos de linfócitos intra-acinares. A presença de necrose em saca bocado ou o comprometimento generalizado dos espaços-porta são critérios de exclusão para esse diagnóstico.

\section{RESULTADOS}

A população estudada foi constituída por 39 pacientes, sendo 23 do sexo masculino (59\%). A idade dos pacientes variou entre 18 e 73 anos,

\begin{tabular}{|lc|}
\hline $\begin{array}{l}\text { Tabela } 3 \text { - Discordância entre diagnóstico clínico (pré- } \\
\text { biópsia) e histopatológico (pós-biópsia) }(\mathbf{n}=14)\end{array}$ \\
\hline $\begin{array}{c}\text { Diagnóstico pré-biópsia } \\
\text { (no- pacientes) }\end{array}$ & $\begin{array}{c}\text { Diagnóstico pós-biópsia } \\
\text { ( } \mathbf{n} \text { - pacientes) }\end{array}$ \\
Hepatite crônica (7) & Hepatite mínima (4) \\
& Cirrose (2) \\
& Normal (1) \\
Cirrose (6) & Normal (2) \\
& Esteatoepatite (2) \\
& Hepatite mínima (1) \\
& Colestase (1) \\
Hepatite por citomegalovírus (1) & Hepatite mínima (1) \\
\hline
\end{tabular}

apresentando média e desvio padrão de 43,7 e 12,2 anos, respectivamente. As indicações para BHTJ nos 39 pacientes aparecem na Tabela 1.

Dos 13 pacientes $(33,3 \%)$ com suspeita de hepatite crônica viral (aminotransferases persistentemente elevadas por mais de seis meses), seis tinham coagulopatia (plaquetopenia em três e tempo de protrombina prolongada em outros três); sete eram renais crôni cos em hemodiálise com uso regular de heparina e anemia crônica com hematócrito igual ou inferior a $29 \%$.

Trinta e nove pacientes foram submetidos a 42 BHTJ . Em dois, o procedimento inicial não obteve sucesso e, posteriormente, foram repetidos, pois a biópsia inicial foi inconclusiva. Em outro, o procedimento foi suspenso devido a formação de grande hematoma cervical por punção acidental da carótida, mas repetido em outra ocasião, com sucesso. Esse paciente, portador de insuficiência renal crônica, havia recebido, inadvertidamente, medicação anticoagulante (heparina) para hemodiálise no dia do exame.

U sou-se sedação intravenosa em citrato de fentanila (50 a $200 \mathrm{mg}$ ) em todos os pacientes. Diazepam foi associado em cinco pacientes (5 a $10 \mathrm{mg}$ ). E m um paciente foi associado midazol an ( $2 \mathrm{mg}$ ); em outro, droperidol ( $5 \mathrm{mg}$ ) e citrato de fentanila (100 mg).

\section{DIAGNÓSTICOS HISTOPATOLÓGICOS}

Material de 36 dos 39 pacientes submetidos a BHTJ foi enviado para estudo histopatológico. O material foi satisfatório e permitiu o diagnóstico em 25 pacientes $(64,1 \%)$. Houve concordância entre o diagnóstico clínico (pré-biópsia) e o diagnóstico histopatológico (pós-biópsia) em 11 pacientes (28,2\%). Em 14 (35,9\%) houve discordância.

Ao revisarmos os prontuários e biópsias dos nove pacientes que apresentaram resultado histopatológico "hepatite mínima" $(n=6)$ ou "normal" $(n=3)$, a média dos tamanhos dos espécimens foi de 
$0,5 \mathrm{~cm}$ na maior dimensão. $O$ número médio de espaços-porta por espécimen foi de 3,6, considerado como suficiente para o diagnóstico pelo patologista. Dentre esses nove indivíduos, cinco eram renais crônicos em hemodiálise (dois com marcadores para hepatite viral $B$; dois para hepatite viral C; e 1 para B e C, simultaneamente); um era recipiente de transplante de medula óssea, com el evação recente nos anticorpos para citomegalovírus, apresentando-se com plaquetopenia $(15.000 / \mathrm{mm} 3)$ e hematócrito abaixo de $29 \%$. Dois pacientes com diagnóstico clínico de hepatite crônica viral possuíam marcadores para hepatite viral $\mathrm{C}$ e, em ambos, havia plaquetopenia. No nono e último paciente, também com marcadores positivos para hepatite viral $C$, o tempo de protrombina era prolongado (abaixo de $50 \%$ do controle em três ocasiões) e havia obesidade mórbida.

\section{Insucessos}

E m sete pacientes (17,9\%), as amostras hepáticas pequenas não permitiram diagnóstico histopatológico conclusivo (diagnósticos clínicos: cirrose em três; hepatite crônica em dois; hepatite crônica e síndrome de Budd-Chiari em um; e tumor metastático infiltrativo em um). Entre sete pacientes $(17,9 \%)$ com diagnóstico clínico de provável cirrose, não foi obtido tecido hepático em quatro (o material continha apenas coágulos); para três houve dificuldades técnicas (impossibilidade de punção da veia jugular em dois e não cateterização da veia hepática em um).

\section{Análise dos espécimens de tecido hepático}

Dentre os 32 pacientes submetidos à BHTJ em que se obteve material hepático, em quatro o material foi fragmentado. Foi possível medir o espécimen em 28, obtendo-se diagnóstico em 25. O comprimento do espécimen apresentou média de $0,55 \mathrm{~cm}(\mathrm{DP}=0,32 \mathrm{~cm})$. Não houve diferença estatística entre os cirróticos e os não cirróticos ( $Z$ = 1,866, $p=0,062 /$ WMW).

\section{Número de espaços-porta}

Dos 25 pacientes, cujo material permitia diagnóstico histopatológico, em 21 foi possível determinar o número de espaços-porta. Nesses indivíduos, o número de espaços-porta variou de 1 a 12 (médi a de 3,6 e mediana de 4,0). E m seis pacientes com diagnósti co histopatológico de cirrose, a avaliação dos espaços-porta ficou prejudicada devido à desestruturação do fígado.

\section{Complicações}

Dor cervical leve e transitória não requerendo analgesia, foi referida por seis pacientes que tive- ram média de 3,1 punções cervicais, superior à média do grupo todo, que foi de 2,7. Em dois deles, ocorreu punção da artéria carótida. Outros cinco pacientes que tiveram a carótida puncionada inadvertidamente não apresentaram sintomas. Houve hematoma cervical em oito pacientes (seis em grau leve; um moderado e um acentuado). Sangramento leve no local da punção, proveniente de pequena veia do tecido subcutâneo, foi observado em um paciente. Perfuração da cápsula hepática ocorreu em três pacientes. Um deles apresentou, após o procedimento, moderada dor abdominal, controlada por anal gesia (petidina intravenosa, $30 \mathrm{mg}$ ); os outros dois permaneceram assintomáticos.

Perfuração de veia calibrosa foi a complicação grave que ocorreu em um paciente. Não foi possível cateterizar a veia hepática e houve cateterização inadvertida da veia renal direita com perfuração da mesma. E sse paciente apresentou acentuada dor abdomi nal, formação de grande hematoma retroperitoneal detectado por ecografia e choque hipovolêmico, sendo necessária intervenção cirúrgica imediata. Durante o ato cirúrgico, foi realizada a biópsia hepática. O paciente está bem e se encontra em tratamento ambulatorial para linfoma não-Hodgkin. Dor abdominal leve e transitória foi verificada em três pacientes durante a introdução da agulha de biópsia na veia hepática e/ou durante a biópsia; entretanto, não houve evidências de complicações ou necessidade de analgesia.

\section{DISCUSSÃO}

A pesar dos avanços nos métodos de investigação das doenças hepáticas, incluindo-se os de imagem e os da bi ol ogia molecular, o exame histopatológi co do tecido hepático é essencial para o diagnóstico e/ ou estadiamento, especialmente nas hepatopatias crônicas. Além da análise histopatológica, a mensuração de metais pesados (como ferro e cobre), de atividades enzimáticas ou a cultura para demonstração de fungos, bactérias e protozoários podem ser de extremo valor no diagnóstico e na conduta terapêutica?.

A biópsia hepática é obtida mais freqüentemente através da via percutânea, mas existem várias situações em que é contra-indicada; uma das vias alternativas é a abordagem transjugular. Há autores que classificam as indicações de BHTJ em maiores ou absolutas e menores ou relativas $^{11,15}$. As mai ores são os distúrbios de coagulação, a ascite maciça e a necessidade de realização simultânea de outro procedimento por via transjugular. Entre as indicações menores estão a obe- 
sidade mórbida, o fígado cirrótico, diminuído de volume, e a necessidade de múltiplas amostras. Há diferença na literatura quanto ao percentual dessas indicações, em virtude de alguns autores terem como indicação principal para a BHTJ as medidas das pressões nas veias hepáticas, sendo a biópsia objetivo secundário. Pacientes submetidos à transplante hepático passaram a ser um importante grupo que se beneficia da BHTJ como alternativa segura para obtenção de tecido hepático, mesmo no período pós-operatório precoce ${ }^{16,17}$. No grupo estudado por nós, incluímos outra indicação para a BHTJ : pacientes com acentuada anemia por insuficiência renal crônica, heparinizados regularmente, durante sessões de hemodiálise, com risco potencial para sangramento e que aguardavam avaliação hepática completa para entrarem em programa de transplante renal.

Coagulopatia esteve presente na maioria dos pacientes estudados, ou seja, 32/39 (82\%), associada com ascite em 17 (43,6\%) e isolada em 13 (33,3\%); na literatura esses achados representam 5-35\% e 50-80\%, respectivamente, das indicações da BHTJ ${ }^{15}$. Entre os dois indi víduos morbidamente obesos, um (2,6\%) apresentava-se com coagulopatia e outro (2,6\%) coagulopatia e ascite maciça.

Em nossa casuística, punções hepáticas foram realizadas em 38 ocasiões, com variação de 1 a 6 punções por procedimento. A literatura não sugere número de biópsias a serem obtidas. Preconizase, como critério, a qualidade do espécimen de fígado obtida. O número máximo de biópsias descrito na experiência de Steadman et al. ${ }^{18}$ foi 10 , e 9 na de $\mathrm{F}$ uruya et al. ${ }^{19}$. Estes últimos autores não vêem relação entre o número de punções biópsias e incidência de complicações.

A duração dos nossos procedimentos para a BHTJ variou de 20 a 120 minutos (média de 56 minutos por exame) ${ }^{20,21}$. Entretanto, o tempo necessário para a realização da BHTJ diminuiu gradualmente no decorrer da série. Isso corrobora a opinião de $\operatorname{Lebrec}^{10}$, de que o procedimento pode levar apenas 10 minutos quando realizado por profissionais experientes. Outros autores referem que o tempo médio necessário é de aproximadamente 30 minutos $^{11,22}$. As dimensões dos espécimens hepáticos obtidos foram semel hantes àquelas referi das na literatura, embora al guns autores tenham encontrado dimensões significativamente maiores com a agulha tTrucut ${ }^{23}$. É importante observar que entre os 20 pacientes com suspeita clínica de cirrose, em 10 não se obteve diagnóstico histopatológico (quatro por material insuficiente; três por não obtenção de teci do hepático; dois por falha na punção da veia jugular; e um por impos- sibilidade de cateterizar a veia hepática direita). Entre os outros 19 diagnósticos sem evidência clínica de cirrose, obteve-se tecido hepático suficiente para diagnóstico em 15. Dos 25 pacientes em que foi obtido o diagnóstico histopatológico (seis com cirrose), a amostra foi fragmentada em um (16,6\%). Em 19 outros sem cirrose o espécimen foi fragmentado em dois indivíduos (10,5\%). Em cinco casos com diagnóstico de cirrose, a amostra foi fragmentada em um (20\%). Apesar da nossa amostra ser pequena, os dados confirmam os relatos da literatura, de que os espécimens de biópsias de pacientes com fibrose hepática e/ou cirrose tendem a ser menores e fragmentados e que, freqüentemente, não permitem o diagnóstico ${ }^{10,11,24-27}$. Face a este fato, tornam-se rel evantes a participação de patologista experiente na análise de tecido hepático e a cautela na sua interpretação ${ }^{27,28}$. $\mathrm{Na}$ série de Lebrec et al. ${ }^{10}$, o material de biópsia permitiu diagnóstico em $64,2 \%$ dos pacientes com fibrose ou cirrose e, nos casos sem fibrose, em $98,9 \%$. Bories et al. ${ }^{29}$ publicaram os resultados de BHTJ em 333 pacientes com cirrose, sendo o diagnóstico al cançado em 249 (77\%).

Conforme Hong-Chiang et al., ${ }^{26}$ a fragmentação do espéci men poderia não ter relação com o calibre da agulha porque seu diâmetro era o mesmo, tanto nas BHTJ quanto nas BHP. Colapinto ${ }^{27,30}$ e Gilmore et al. ${ }^{8}$ preconizam o uso de estilete tipo Menghini dentro da agulha de biópsia para impedir possível aspiração e fragmentação do espécimen no interior da seringa. Schiff et al. ${ }^{31}$ referem que a fragmentação é mais provável com a técnica de Menghini e, segundo outros autores, a agulha Trucut deve ser usada se houver suspeita de cirro$\mathrm{se}^{23}$. Colapinto ${ }^{27}$ preconiza o uso de agulha Trucut, pois esta oferece amostras maiores e menos fragmentadas, tornando o procedimento mais fácil.

No grupo de 39 pacientes submeti dos à $\mathrm{BHTJ}$ na nossa série, obteve-se tecido hepático em 32 (82,1\%). Diagnóstico histopatológico foi obtido em 25 (64,1\%). O resultado histopatológico confirmou o diagnóstico clínico (pré-biópsia) em 11 pacientes (28,2\%). Houve discordância em 14 (35,9\%), notavel mente no grupo de seis indi víduos com suspeita clínica de cirrose. É provável que tenha havido erro amostral significativo neste grupo, especialmente quando se considera a existência de tecido hepático sem fibrose e aspecto histopatológico "normal" em dois e "hepatite mínima" em um desses indivíduos. Surpreendentemente, em nove indivíduos, todos com el evação leve mas persistente, nas aminotransferases e marcadores virais positivos, o diagnóstico foi "normal" ou "hepatite mínima", sendo cinco portadores de insuficiência 


\begin{tabular}{|c|c|c|c|c|}
\hline Referência & Localidade & $\begin{array}{l}\text { Procedimentos } \\
\text { (n) }\end{array}$ & $\begin{array}{l}\text { Obtenção de } \\
\text { tecido hepático(n) }\end{array}$ & $\begin{array}{c}\text { Obtenção de } \\
\text { diagnóstico (n) }\end{array}$ \\
\hline Rösch et al. ,1975 & Portland, EUA & 83 & 79 (95\%) & $71(86 \%)$ \\
\hline Gilmore et al., 1977 & Londres, Inglaterra & 26 & $26(100 \%)$ & $26(100 \%)$ \\
\hline Choy et al., 1978 & Bridgeport, EUA & 32 & $31(97 \%)$ & $28(88 \%)$ \\
\hline Goldman et al., 1978 & Atlanta, EUA & 76 & $63(83 \%)$ & $63(100 \%)$ \\
\hline Matzen et al., 1979 & Copenhage, Dinamarca & 21 & $17(81 \%)$ & $17(76 \%)$ \\
\hline Lebrec et al., 1982 & Clichy, França & 1033 & $1000(97 \%)$ & 709 (69\%) \\
\hline Maillet et al., 1982 & Lyon, França & 42 & $41(98 \%)$ & $41(98 \%)$ \\
\hline Bull et al., 1983 & London, Inglaterra & 193 & $190(98 \%)$ & $188(99 \%)$ \\
\hline Reding, 1983 & Bruxelas, Bélgica & 100 & 95 (95\%) & 95 (95\%) \\
\hline Bataile \& Bercoff, 1983 & Liège, França & 215 & $213(99 \%)$ & $136(64 \%)$ \\
\hline Velt et al., 1984 & Bridgeport, EUA & 160 & - & $129(81 \%)$ \\
\hline Gamble et al., 1985 & Toronto, Canadá & 461 & $443(96 \%)$ & 425 (92\%) \\
\hline Steadman et al., 1988 & Birbaine, Austrália & 67 & - & $62(93 \%)$ \\
\hline Vlavianos et al., 1991 & London, Inglaterra & 104 & - & $89(87 \%)$ \\
\hline McAfee et al., 1992 & Portland, EUA & 146 & $134(92 \%)$ & $115(79 \%)$ \\
\hline Corr et al., 1992 & Hong-kong, China & 200 & $168(84 \%)$ & $155(77 \%)$ \\
\hline Azoulay et al., 1996 & Paris, França & 124 & $87(70 \%)$ & $86(69 \%)$ \\
\hline Maciel et al., 1996 & Porto Alegre, Brasil & 42 & $32(76 \%)$ & $25(60 \%)$ \\
\hline
\end{tabular}

renal crônica em avaliação para transplante renal. Os espécimens hepáticos nesse subgrupo continham uma média e mediana de espaços-porta similar a média do restante do grupo, mas é possível que não tenham sido representativos por um processo inflamatório, provavelmente presente no tecido hepático. Sabemos que biópsias hepáticas representam um minúsculo fragmento de um órgão vol umoso e, mesmo em doenças que envolvem difusamente o fígado, como as hepatites crônicas virais, podem não ser representativas das alterações histopatológicas presentes ${ }^{32}$. A interpretação dos diagnósticos histopatológi cos foi feita com cautela nos casos discordantes do diagnóstico clínico.

O impacto na terapêutica, qualidade de vida e no prognóstico não foi o propósito deste trabalho. Entretanto, o estabelecimento de diagnóstico histopatológico em $25(25 / 39=64,1 \%)$ dos indivíduos estudados, em geral muito graves e freqüentemente considerados "intocáveis" para biópsia hepática, provavelmente contribuiu para significativas mudanças na conduta clínica. Esses dados reforçam os relatos de vários autores que salientam a importância da obtenção de tecido hepático para o estudo histopatológico. Na série de $\mathrm{F}$ uruya et al . ${ }^{19}$ esse fato ocorreu em $30 \%$ dos pacientes e em
$31 \%$ nas séries de Velt et al. ${ }^{33}$ e Witt-Sullivan et al. ${ }^{34}$. Das 124 BHTJ realizadas por MCAfee et al. ${ }^{11}$, $11,3 \%$ tiveram novo diagnóstico e $21,8 \%$ tiveram mudanças na conduta terapêutica.

A Tabela 4 mostra os índices de sucesso da BHTJ em diversos centros, em comparação com os nossos resultados.

Em nossa série, podemos observar um menor índice de sucesso da BHTJ em relação a outros autores. Contudo, a revisão crítica de um dos artigos mais citados na literatura ${ }^{8}$ revela que tecido hepático suficiente para o diagnóstico foi obtido em apenas $61,5 \%$ dos pacientes, diferindo dos percentuais que diversos autores citam ${ }^{11,15,27,28}$. Esse fato pode ser parcial mente explicado pel o que Steadman et al. ${ }^{18}$ chamam de curva de aprendizado. Acreditamos que a experiência obtida pela continuidade na realização de procedimentos de BHTJ certamente permitirá obter resultados diagnósticos mais significativos e, provavel mente, com menor índice de complicações. A tendência de as amostras obtidas pela BHTJ serem menores e mais fragmentadas, pode ser revertida com o uso da agulha Trucut 11,26 , hoje disponível no país e já utilizada por nós para a abordagem transjugular. A BHTJ é melhor realizada por radiologista com 


\begin{tabular}{|c|c|c|c|c|c|c|c|c|}
\hline Autores & $\begin{array}{c}\text { Dor } \\
\text { cervical }\end{array}$ & $\begin{array}{l}\text { Sangramento } \\
\text { vaso cervical }\end{array}$ & $\begin{array}{l}\text { Hematoma } \\
\text { cervical }\end{array}$ & $\begin{array}{l}\text { Arritmias e } \\
\text { taquicardias supra } \\
\text { ventriculares }\end{array}$ & $\begin{array}{c}\text { Dor } \\
\text { abdominal }\end{array}$ & $\begin{array}{l}\text { Perfuração } \\
\text { grande vaso }\end{array}$ & $\begin{array}{l}\text { Perfuração } \\
\text { cápsula }\end{array}$ & Morte \\
\hline $\begin{array}{l}\text { Lebrec et al. } 1982 \\
\text { (932 pacientes) }\end{array}$ & - & - & $10(1,1 \%)$ & $6(0,6 \%)$ & $74(7,9 \%)$ & - & $5(0,5 \%)$ & $1(0,1 \%)$ \\
\hline Bull et al. 1983 & - & $2(1,0 \%)$ & - & $5(2,6 \%)$ & - & - & $2(0,2 \%)$ & $1(0,5 \%)$ \\
\hline $\begin{array}{l}\text { Gamble et al. } 1985 \\
\text { (436 pacientes) }\end{array}$ & $7(1,6 \%)$ & $8(1,8 \%)$ & $17(3,9 \%)$ & $2(05 \%)$ & $7(1,6 \%)$ & - & $18(4,1 \%)$ & $1(0,2 \%)$ \\
\hline $\begin{array}{l}\text { Vlavianos et al. } 1991 \\
\text { (104 pacientes) }\end{array}$ & - & - & - & $3(2,9 \%)$ & - & - & $6(5,8 \%)$ & $1(1,0 \%)$ \\
\hline $\begin{array}{l}\text { McAfee et al. } 1992 \\
\text { (140 pacientes) }\end{array}$ & - & $5(3,6 \%)$ & $5(3,6 \%)$ & $2(1,4 \%)$ & $5(3,6 \%)$ & - & $2(1,4 \%)$ & - \\
\hline $\begin{array}{l}\text { Corr et al. } 1992 \\
\text { (200 pacientes) }\end{array}$ & - & - & - & $3(1,5 \%)$ & - & - & $12(6,0 \%)$ & - \\
\hline $\begin{array}{l}\text { Furuya et al. } 1992 \\
\text { (27 pacientes) }\end{array}$ & - & - & - & - & - & 1 (VCI) $(3,7 \%)$ & - & - \\
\hline $\begin{array}{l}\text { Donaldson et al. } \\
1993 \text { (61 pacientes) }\end{array}$ & - & $2(3,3 \%)$ & - & - & - & 1 (VCI) $(1,6 \%)$ & - & - \\
\hline $\begin{array}{l}\text { Maciel et al. } 1996 \\
\text { (39 pacientes) }\end{array}$ & $6(15,4 \%)$ & $1(2,6 \%)$ & - & $3(7,7 \%)$ & $\begin{array}{c}1 \text { (veia renal } \\
\text { direita) }(2,6 \%)\end{array}$ & $3(7,7 \%)$ & - & \\
\hline
\end{tabular}

experiência em cateterização da veia hepática e é um procedimento essencial em serviços de hepatologi $a^{10}$. Os radiologistas envolvidos nessa técnica não a têm considerado como método difícil, embora a BHTJ seja mais complexa do que a BHP. Além de exigir profissionais treinados em técnica angiográfica, a BHTJ é mais demorada, necessita de equipamentos de hemodinâmi ca e el etrocardiografia e requer hospitalização, o que torna este procedimento mais oneroso do que a abordagem percutânea ${ }^{18}$.

A Tabela 5 mostra as complicações encontradas nesta investigação, comparadas aos resultados de outros autores na literatura.

Steadman et al. ${ }^{18}$ salientaram que comparações entre vários tipos de biópsia relativas aos percentuais de complicações referidas por diferentes autores precisam ser analisadas com prudência, visto que há critérios muito diferentes para definilas. Optamos por critérios rigorosos, considerando, por exemplo, qualquer intensidade no sintoma "dor" como relevante. Em muitos trabalhos, sintomas menores não foram sequer tabulados.

Taquicardia supraventricular paroxística ou extra-sistolia persistentes não foram vistas em nossa casuística, mas foram observadas por Lebrec em seis de 932 pacientes $(0,6 \%)^{10}$. Entretanto, na grande maioria dos nossos pacientes, houve breve extra-sistolia ventricular quando ocorria penetração do fio guia no ventrículo direito, pron- tamente reversível tão logo fosse corrigido o curso do fio guia.

A ocorrência de perfuração de grandes vasos é pouco freqüente na literatura e raros óbitos são relatados ${ }^{10,23,34-37}$. A perfuração da veia renal direita que ocorreu em um dos nossos pacientes foi a única complicação grave em nossa experiência, sendo o paciente submeti do a intervenção cirúrgica imediata e exitosa ${ }^{20,21,38}$.

A perfuração da cápsula hepática, por outro lado, é freqüente e ocorre em 0,5 a $5,8 \%$ das séries na literatura, principalmente em pacientes com fígados cirróticos de pequeno volume. É considerada potencialmente grave pelo risco de hemorragia intra-abdominal ${ }^{10,11,20,23,24,35-39}$. Surpreendentemente, a literatura indica que a grande maioria dos pacientes permanecem assintomáticos, mas recomenda a injeção rotineira de contraste após a realização da BHTJ para não subestimar a incidência de perfuração capsular ${ }^{23}$. Nós tivemos a perfuração da cápsula do fígado em três pacientes $(7,7 \%)$; em um, verificou-se dor abdominal moderada controlada com petidina IV, enquanto nos outros dois não se observaram quaisquer sintomas ou al terações hemodinâmicas.

A B HP continuará a ser o método preferido para o estudo do tecido hepático. Porém, a BHTJ permite a um grupo de pacientes particularmente graves (com coagulopatia importante e/ou ascite maciça e anemia crônica acentuada associada à insu- 
ficiência renal), principalmente em vigência de tratamento com heparina, a possibilidade de diagnóstico histopatológico com eventual instituição ou mudança na conduta terapêutica.

\section{CONCLUSÕES}

1. Em 25 dos 39 pacientes $(64,1 \%)$ a BHTJ permitiu obtenção de tecido hepáti co satisfatório e de diagnóstico histopatológico. Houve dificuldade na obtenção de tecido hepático e, provavelmente, signifi cativo erro amostral no grupo com suspeita clínica de cirrose. É provável que esses fatos se devam à utilização da agulha de Menghini no estudo. A introdução da agulha de trucut e a experiência crescente dos autores deverão el evar, progressivamente, o índice de sucesso.

2. Apesar das complicações da BHTJ, esta foi bem tolerada pela maioria dos pacientes, mesmo no período inicial de desenvolvimento desta técnica em nosso serviço.

3. A BHTJ é um método diagnóstico útil para obtenção de tecido hepático em pacientes com suspeita de hepatopatia crônica e contra-indicação à $\mathrm{BHP}$; entretanto é relativamente complexa, requer treinamento sofisticado e deve ser reservada a centros de excel ência em hepatologia.

\section{SUMMARY}

Transjugular liver biopsy: experience in patients with suspected chronic liver disease and contraindication for percutaneous liver biopsy using modified Ross needle

Purpose: This work aimed at introducing the transjugular liver biopsy technique oriented by radiologic methods in our hospitals. This technique is indicated for obtaining histopathologic diagnosis in patients clinically and laboratorially suspected of having chronic liver disease with contraindications to percutaneous liver biopsy.

Methods: Liver biopsy was obtained with a modified Ross needle through the right jugular vein and right hepatic vein under fluoroscopic control.

Results: Transjugular liver biopsy was attempted for 39 patients, liver tissue obtained en 32 and histopathologic diagnosis in 25 (64.1\%). In 11 patients $(28.2 \%)$ there was agreement between the diagnoses established before and after biopsy, however, in 14 patients (35.9\%), there was disagreement. The yield of diagnosis was low when patients were suspected for cirrhosis. The proce dure was well tol erated by the majority of patients. Nonetheless, 1 presented intra-abdominal blee ding and required immediate surgery to control retroperitoneal hemorrhage.

Conclusions: Transjugular liver biopsy is useful for the histopathologic diagnosis of patients with chronic liver diseases whenever the percutaneous route is contraindicated. In this series we obtained histopathologic diagnosis for $64,1 \%$ of the subjects studied. Patients suspected of having cirrhosis had a low yield of histopathologic diagnosis (50\%) when compared to subjects without clinical evidence for crrhosis (78,9\%). The technique is rather complex, and can cause serious complications. This, it should be performed in reference centers in radiology and hepatology. [Rev Ass Med Bras 2000; 46(2): 134-42]

KEY WORDS: Liver biopsy. Transjugular liver biopsy. Liver. Coagulopathy. Chronic liver diseases. Diagnosis.

\section{Nota}

* Parte deste trabalho origina-se da Dissertação de Mestrado do primeiro autor (ACM), apresentada publicamente e aprovada com grau "A" pela banca examinadora constituída pelos Professores Hilton Augusto Koch (Universidade Federal do Rio de Janeiro), Cleber Dario Pinto Kruel e Luís Pereira Lima (Universidade Federal do Rio Grande do Sul) ao Curso de Pós-graduação em Medicina: Gastroenterologia, para obtenção do título de Mestre em Medicina - Faculdade de Medicina - Universidade Federal do Rio Grande do Sul. Porto Alegre, 1996.

\section{REFERÊ NCIAS BIBLIOGRÁFICAS}

1. Babb RR, J ackman RJ . Needle biopsy of the liver: a critique of four currently available methods. WestJ Med 1989; 150: 39-42.

2. Dotter CT. Catheter biopsy. Experimental technic for transvenous liver biopsy. Radiology 1964; 82: 312-14.

3. Hanafee W, Weiner M. Transjugular percutaneous cholangiography. Radiology 1967; 88: 35-9.

4. Weiner M, Hanafee WN. A review of transjugular cholangiography. Radiol Clin North Am 1970; 8: 53-68.

5. Rösch J , Lakin PC, Antonovic R et al. Transjugular approach to liver biopsy and transhepatic cholangiography. N Engl J Med 1973; 289: 227-31.

6. Rösch J , Lakin PC, AntonovicR et al. Transjugular liver biopsy and cholangiography. Fortschr Röntgenstr 1973; 119: 652-61.

7. Sherlock S, Dick R, Van Leenwen DJ . Liver biopsy today. The Royal Free Hospital experience. J Hepatol 1984; 1: 75-85.

8. Gilmore IT, Bradley RD, Thompson RPH. I mproved method of transvenous liver biopsy. Br Med J 1977; 2: 100-1.

9. Poorter J D. La biopsie hépatique trans-veineuse par voie transjugulaire. Soins T 1980; 25: 223-5.

10. Lebrec D, Goldfarb G, Degott C, Rueff B, Benhamou J . Transvenous liver biopsy. Gastroenterol ogy 1982; 83: 338-40.

11. McAfee J H, Keefee EB, Lee RG, Rösch J. Transjugular liver biopsy. Hepatology 1992; 15: 726-32.

12. Menghini G. One-second needle biopsy of the liver Gastroenterol ogy 1958; 35: 190-9.

13. Zar J H. Biostatistical Analysis, 2 ed. New J ersey, PrenticeHall, 1984.

14. Bianchi L, Gudat F. Chronic hepatitis. In MacSween RNM, 
Anthony PP, Scheuer PJ et al. Eds. Pathology of theliver. $3^{\text {rd }}$ ed. Edinburg: Churchill Livingstone; 1994.

15. Zwiebel FM, Holl J , Kleber G. Transjuguläre Leberpunktion. Bildgebung 1993; 60: 161-8.

16. Azoulay D, Raccuia J S, Roche B, Reynes M, Bismuth H. The value of early transjugular liver biopsy after liver transplantation. Transplantation 1996; 61(3): 406-9.

17. Lebrec D. Various approaches to obtaining liver tissue choosing the biopsy technique. J Hepatol 1996; 1: 20-4.

18. Steadman C, TeagueC, Harper J et al. Transjugular liver biopsy - An Australian experience. Aust NZJ Med 1988; 18: 836-40.

19. Furuya KN, Burrows PE, Phillips MJ et al. Transjugular liver biopsy in children. Hepatol ogy 1992; 15: 1.036-42.

20. Maciel AC. Biópsia hepática transjugular: experiência inicial com 39 pacientes. Dissertação de Mestrado. Porto Alegre: Universidade F ederal do Rio Grande do Sul; 1996.

21. Maciel AC, J unior LCVS, Barros SGS et al. Biópsia hepática transjugular: contribui ção do radi ol ogista para o diagnóstico histopatológi co em hepatopatas com distúrbios de coagulação e/ou ascite. Radiol Bras 1998; 31: 65-74.

22. Cañizares RB, Ricote GC, Castro LS et al. Biopsia hepática transyugular: A propósito delos cien primeros casos y revisión de la literatura. Rev Esp E nf Digest 1992; 81: 185-8.

23. Bull HJ M, Gilmore IT, Bradley RD et al. Experience with transjugular liver biopsy. Gut 1983; 24: 1.057-60.

24. Corr P, Beningfield SJ , Davey N. Transjugular liver biopsy: a review of 200 biopsies. Clin Radiol 1992; 45: 238-9.

25. Sherlock S. Needle biopsy of the liver. In Sherlock S, Doodley J eds. Diseases of theliver and biliary system, 9th ed. Oxford, Blackwell Scientific Publications, 1993; 36-48.

26. Hong-Chiang $M$, Han-Chieg $L$, ChengChun $H$ et al. Transjugular liver biopsy: comparison with percutaneous liver biopsy. J Gastroenterol Hepatol 1994; 9: 457-61.

27. Colapinto RF. Transjugular biopsy of the liver. Clin Gas- troenterol 1985; 14: 151-67.

28. J ackson J E, Adam A, Allison DJ . Transjugular and plugged liver biopsies. Baillière's Clin Gastroenterol 1992; 6: 2.245-58.

29. Bories $P$, Chaker W, Mirouze $D$ et al. Intérêt de l'abord veineux transjugulaire du foie dans le diagnostic de la cirrhose. La Presse Médicale 1985; 14: 1.543-5.

30. Colapinto RF, Blendis LM. Liver biopsy through the transjugular approach. Modifications of instruments. Radiology 1983; 148: 306.

31. Schiff ER, Schiff L. Needle biopsy of the liver. In Schiff L, Schiff EL eds. Diseases of the liver, 7th ed. Philadelphia, Lippincott, 1993; 216-25.

32. Maharaj B, Leary WP, Naran AD et al. Sampling variability and its influence on the diagnostic yield of percutaneous needle biopsy of the liver. Lancet 1986; 1: 523-5.

33. Velt PM, Choy OG, Shimkin PM et al. Transjugular liver biopsy in high-risk patients with hepatic disease. Radiology 1984; 153: 91-3.

34. Witt-Sullivan $\mathrm{H}$, HeathcoteJ , Simons $\mathrm{M}$ et al. Clinical usefulness of the transjugular liver biopsy. Hepatology 1990; 12: 746.

35. Mal F, Meyrier A, Callard $P$ et al. Transjugular renal biopsy. Lancet 1990; 335: 1.512-13.

36. GambleP, Colapinto RF, Stronell RD et al. Transjugular liver biopsy: a review of 461 biopsies. Radiology 1985; 157: 589-93.

37. Vlavianos P, Bird G, Portmann B et al. Transjugular liver biopsy: use in a selected high- risk population. Eur J Gastroenterol Hepatol 1991; 3: 469-72.

38. Maciel AC, Barros SGS, Cerski CTS et al. A biópsia hepática transjugular: uma abordagem para diagnóstico na doença hepática com coagulopatia. Revista HCPA 1997; 17: 105-8.

39. Goldman ML, Gonzales AC, Galambos J T et al. The transjugular technique of hepatic venography and biopsy, cholangiography, and obliteration of esophageal varices. Radiology 1978; 1.228: 3225-31. 